\title{
The Relation between Blood Platelet Count and Arakawa's Reaction in Lactating Mothers Taking Vitamin B.*
}

I32nd Report of the Peroxidase Reaction.

By

Shizuo Kimura.

(木 朴 翡 椎)

(From the Department of Pediatrics, Faculty of Medicine, Tohoku Imperial University, Sendai.

Director: Prof. A. Sato.)

\section{Introduction.}

In a preceding paper ${ }^{1)}$ I reported that most of apparently healthy mothers secreting milk negative to A rakaw a's reaction** showed a high blood platelet count; and was able to confirm J. Kimura's $\mathrm{s}^{2-4)}$ results.

The cases I reported in the preceding paper were not taking any vitamin B preparation. Then, how about mothers with negative A rakaw a's reaction taking it? In the present paper the relationship between the blood platelet count and A rakawa's reaction in the case of lactating mothers taking vitamin $B$ preparations will related.

* $B_{1}$ is meant throughout the article.

** "Arakawa-positive" may be used in two different senses. One of these is: Arakawa-positive in a biochemical sense. A sample of human milk is said to have become Arakawa-positive, when it became blue on the addition of Arakawa's reagent. Here it means that the sample was not negative to A rakawa's reastion. The other of these two senses is: Arakawa-positive in a clinical sense. A sample of haman milk is clinically Arakawa-positive only when it shows such a reaction as \# or $\#$ in one minute of the addition of Arakawa's reagent. Another sample of human milk may be Arakawa-positive in the first deseribed sense, but yet clinieally negative.

1) Sh. Ki mura, Tohoku J. Exp. Med., 1939, 37, 241.

2) J. Kimura, Tohokr J. Exp. Med, 1934, 23, 494.

3) J. Kimura, Tohoku J. Exp. Méd., 1935, 27, 360.

4) J. Kimura, Tohoku J. Exp. Med., 1935, 27, 374. 


\section{Method of Investigation.}

1. Materials. Blood was taken from apparently healthy mothers of breastfed patients who had long been taking vitamin $B$ preprations although they were not suffering from beriberi. But the amounts and and the kinds of vitamin B preparations which were taken by these mothers were very different, and how long they had been taking them was not clear.

2. Arakawa's reaction.

a. Milk was examined with A rakawa's reagent in each case.

b. I divided all the cases into 4 groups according to the intensity of Arakawa's reaction as in my preceding paper ${ }^{1)}$. Group I was the only group which ought to be considered as normal as to the reaction in a clinical sense, Group II was a group of Arakawa-negative case (or case with better intermediate A ra ka w a's reaction), group IIr was a group of Arakawa-negative case (or case with worse intermediate Arakawa's reaction) and Group IV was a group of completely or almost completely Arakawa-negative case (Cf. Table 1).

TABLE 1.

Grouping of cases according to the intensity of A rakawa's reaction of human milk.

\begin{tabular}{|c|c|c|c|}
\hline \multirow{3}{*}{ Group } & \multicolumn{3}{|c|}{ Intensity of Arakawa's reaction of human milk } \\
\hline & \multirow{2}{*}{$\begin{array}{l}\text { Arakawa-positive or -negative } \\
\text { in clinical sense }\end{array}$} & \multicolumn{2}{|c|}{ Expressed in signs } \\
\hline & & one side & the other side \\
\hline $\mathbf{I}$ & Arakawa-positive case (normal case) & $\begin{array}{l}\text { (Ht) } \mathbf{1}^{\prime} \\
(H) \mathbf{1}^{\prime}\end{array}$ & $\left\{\begin{array}{l}(+4) \\
(+H) \\
(+H) \\
1^{\prime}\end{array}\right.$ \\
\hline II & $\begin{array}{l}\text { Arakawa-negative case (or case with better } \\
\text { intermediate Araka wa's reaction) }\end{array}$ & $\begin{array}{l}\text { (H) } x^{\prime} \\
(H) 1^{\prime} \\
(+) 1^{\prime}\end{array}$ & $\left\{\begin{array}{l}( \pm) x^{\prime} \text { or }(-) x^{\prime} x^{\prime} \\
(+) 1^{\prime}, \quad( \pm) 1^{\prime} \\
\text { or }(-) 1^{\prime} \\
( \pm) 1^{\prime}\end{array}\right.$ \\
\hline III & $\begin{array}{l}\text { Arakawa-negative case (or case with worse } \\
\text { intermediate Araka wa's reaction) }\end{array}$ & $\begin{array}{l}(+) 1^{\prime} \\
\pm \\
(-) \\
1^{\prime}\end{array}$ & 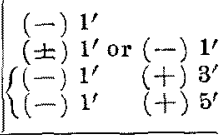 \\
\hline IV & $\begin{array}{l}\text { Completely or almost completely A } \mathrm{rak} \text { a wa- } \\
\text { negative case }\end{array}$ & $\begin{array}{l}(-) 5 \\
(-) 5\end{array}$ & $\begin{array}{l}( \pm) 5^{\prime} \\
(-) 5^{\prime}\end{array}$ \\
\hline
\end{tabular}


3. Platelet count. I used Lampert's $\mathbf{s}^{5)}$ method. The ear lobe was cleaned with ether, and a cut was made with Francke's needle. Blood was taken into the "Athrombit" and diluted with Tyrode's solution. After shaking the pipette for a few minutes, the 6th drop of the contents was examined for blood platelets. I waited over 5 minutes after the counting chamber was filled, and counted only then. Blood taking took place between 11 and 12 o'clock a.m., when mothers were in a fasting state.

I classified all the cases into 4 groups according to their platelet count: the group with "no increase" (or with the platelet count of 200-400 thousands), the group with "slight increase" (or with the platelet count of 400-500 thousands), the group with "moderate increase" (or with the platelet count of 500-600 thousands), and the group with "remarkable increase" (or with the platelet count of over 600 thousands).

\section{Results of Investigation.}

109 cases were examined and the results were as shown in Table 2. All of them had been taking a certain vitamin $B$ preparation on their doctor's advice because of their Arakawa-negative milk (Cf. Table 2). and 3 ).

A) Classification according to the platelet count (Cf. Tables 2

1. Cases with no increase: There were 31 cases out of 109 cases that showed no increase of the platelet count. 7 cases out of them belonged to Group I of A raka wa's reaction, 4 cases to Group II, 11 cases to Group III and 9 cases to Grup IV.

2. Cases with a slight increase of the platelet count: In 61 cases out of 109 cases the count showed a slight increase. Out of them 1 case belonged to Group I of A rakawa's reaction, 5 cases to Group II, 26 cases to Group III and 29 cases to Group IV.

3. Cases with a moderate increase of the platelet count: In 13 cases out of 109 cases the count showed a moderate increase. There was no case belonging to Group I. 1 case belonged to Group II, 6 cases to Group III and 6 cases to Group IV.

4. Cases with a remarkable increase of the platelet count: Only 4 cases out of 109 cases showed a remarkable increase of the count.

5) H. L a m pert, Verh. Dtsch. Ges. inn. Med., 1931, 43, 92. 
TABLE 2.

Blood platelet Count of mothers with different Arak awa's reaction taking vitamin $B$.

\begin{tabular}{|c|c|c|c|c|c|c|c|c|}
\hline \multirow{2}{*}{$\begin{array}{l}\text { Case } \\
\text { No. }\end{array}$} & \multirow{2}{*}{ Name } & \multirow{2}{*}{$\begin{array}{l}\text { Month } \\
\text { of } \\
\text { experi- } \\
\text { ment }\end{array}$} & \multirow{2}{*}{$\begin{array}{l}\text { Age of } \\
\text { mother } \\
\text { (jears) }\end{array}$} & \multirow{2}{*}{$\begin{array}{l}\text { Age of } \\
\text { infant } \\
\text { (months) }\end{array}$} & \multirow{2}{*}{$\begin{array}{l}\text { Blood plate- } \\
\text { let count } \\
\text { (thousands) } \\
\text { per cmm. }\end{array}$} & \multicolumn{2}{|c|}{$\begin{array}{l}\text { Arakawas } \\
\text { reaction }\end{array}$} & \multirow{2}{*}{$\begin{array}{l}\text { Increase } \\
\text { of blood } \\
\text { platelets }\end{array}$} \\
\hline & & & & & & Right & Left & \\
\hline 1 & Y.S. & June & 32 & 16 & 380 & $1(H)$ & $1(H)$ & \\
\hline 2 & M.M. & July & 25 & 4 & 300 & $1(+)$ & $9+5$ & \\
\hline 3 & K.T. & Aug. & 26 & 2 & 310 & $16(-)$ & $16(-)$ & \\
\hline 4 & S. H. & & 29 & 7 & 320 & $1\left(\frac{n}{t+1}\right)$ & $1(H)$ & \\
\hline 5 & T.M. & Feb. & 28 & 3 & 320 & $3( \pm)$ & $5( \pm)$ & \\
\hline 6 & A.N. & & 28 & 2 & 320 & $16(-)$ & $16(二)$ & \\
\hline 7 & Y.M. & March & 30 & 2 & 320 & $16(-)$ & $16(-)$ & \\
\hline 8 & T.T. & Oct. & 30 & 13 & 330 & $3(+t)$ & $2(H)$ & \\
\hline 9 & H.K. & June & 28 & 10 & 330 & $2(+)$ & $2(+)$ & \\
\hline 10 & M.K. & Dec. & 25 & 2 & 330 & $4(-)$ & $16(-)$ & \\
\hline 11 & Y.N. & Feb. & 23 & 13 & 340 & $2(+)$ & $15( \pm)$ & \\
\hline 12 & K. 0 . & $n$ & 27 & 13 & 340 & $10( \pm)$ & $10( \pm)$ & \\
\hline 13 & A.T. & Aug. & 26 & 9 & 340 & $1( \pm)$ & $16(-)$ & $\underset{d}{d}=F=$ \\
\hline 14 & M.M. & May & 25 & 2 & 340 & $2( \pm)$ & $16(-)$ & 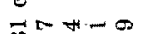 \\
\hline 15 & K. M. & Feb. & 30 & 4 & 340 & $\left.16^{\prime}-\right)$ & $16-5$ & $\infty$ \\
\hline 16 & S. T. & Dec. & 26 & 3 & 350 & $1(H)$ & $1(H)$ & \\
\hline 17 & C. T. & Sep. & 33 & 2 & 360 & $1(H)$ & $1(H)$ & 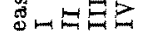 \\
\hline 18 & $\mathrm{~K} . \mathrm{S}$. & Jan. & 27 & 6 & 360 & $16(-)$ & $1( \pm)$ & 可 \\
\hline 19 & T.T. & Oet. & 27 & 10 & 370 & $1(H)$ & $3(\pi)$ & \\
\hline 20 & T.A. & Mareh & 24 & 11 & 370 & $5(+)$ & $1(+)$ & $\circ$ \\
\hline 21 & K.X. & Dee. & 28 & 2 & 370 & $16(-)$ & $16(-)$ & $Z_{1}$ \\
\hline 22 & G.S. & Oct: & 25 & 11 & 380 & $1(+t)$ & $1(H)$ & $b$ \\
\hline 23 & C.I. & April & 33 & 8 & 390 & $4(-)$ & $10(-)$ & \\
\hline 24 & N:S. & June & 31 & 1 & 390 & $16(-)$ & $16(-)$ & \\
\hline 25 & K.S. & Jan. & 35 & 2 & 400 & $1( \pm)$ & $1( \pm)$ & \\
\hline 26 & Y.S. & June & 32 & 16 & 400 & $14( \pm)$ & $16(-)$ & - \\
\hline 27 & M.A. & Jan. & 27 & 1.5 & 400 & $14( \pm)$ & $12(-)$ & \\
\hline 28 & T.A. & Feb. & 28 & 8 & 400 & $2(-)$ & $3( \pm)$ & \\
\hline 29 & I. K. & $\pi$ & 26 & 5 & 400 & $15-$ & $16(-)$ & \\
\hline 30 & U.K. & June & 26 & 1 & 400 & $16(-)$ & $16(-)$ & \\
\hline 31 & M.W. & $n$ & 24 & 1.5 & 400 & $16(-)$ & $16(-)$ & \\
\hline 32 & K.C. & Dee. & 26. & 3 & 410 & $5(++)$ & $11(+)$ & \\
\hline 33 & T.K. & Nov. & 29 & 2 & 410 & $10( \pm)$ & $15 \pm$ & \\
\hline 34 & S. 0 & 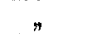 & 21 & 9 & 410 & $3(-)$ & $5( \pm)$ & \\
\hline 35 & H.F. & Aug. & 26 & 5 & 410 & $13(-)$ & $13(-)$ & \\
\hline 36 & J.T. & June & 30 & 10 & 410 & $8(-)$ & $12(-)$ & \\
\hline 37 & R.F." & Oet. & 35 & 14 & 420 & $3(++)$ & $3(H)$ & \\
\hline 38 & F.I. & June & 36 & 10 & 420 & $1(+4)$ & $1( \pm)$ & \\
\hline 39 & M.F. & Feb. & 26 & 0.5 & 420 & $16(-)$ & $14 \pm$ & \\
\hline 40 & J. 0 & Mach & 23 & 1 & 420 & $16(-)$ & $14( \pm)$ & \\
\hline 41 & $\mathrm{~T} . \mathrm{A}$. & Jan. & 27 & 8 & 420 & $7(-)$ & $11(-)$ & \\
\hline 42 & H.E. & March & 38 & 1 & 420 & $15(-)$ & $15(-)$ & \\
\hline 43 & H.K. & Dec. & 26 & 10 & 420 & $16-)$ & $16(-)$ & . \\
\hline 44 & K.O. & April & 30 & 6 & 420 & $16(-)$ & $16(-)$ & \\
\hline 45 & H.O. & Jan. & 28 & 4 & 420 & $16(-)$ & $16(-)$ & \\
\hline 46 & F.K. & June & 31 & 1.5 & 430 & $1(+)$ & $10( \pm)$ & 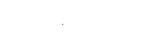 \\
\hline 47. & M.S. & March & 24 & 5 & 430 & 14 (土) & $13 \pm$ & \\
\hline 48 & $\mathrm{Y} . \mathrm{C}$. & May & 25 & 5 & 430 & $16(-)$ & $6(-)$ & \\
\hline 49 & K.S. & July & 31 & 16 & 430 & $14(-)$ & $5( \pm)$ & \\
\hline 50 & S.F. & Jan. & 30 & 4 & 430 & $3( \pm)$ & $2(-)$ & \\
\hline
\end{tabular}




\begin{tabular}{|c|c|c|c|c|c|c|c|c|}
\hline \multirow{2}{*}{$\begin{array}{l}\text { Case } \\
\text { No. }\end{array}$} & \multirow{2}{*}{ Name } & \multirow{2}{*}{$\begin{array}{l}\text { Month } \\
\text { of } \\
\text { experi- } \\
\text { ment }\end{array}$} & \multirow{2}{*}{$\begin{array}{l}\text { Age of } \\
\text { mother } \\
\text { (years) }\end{array}$} & \multirow{2}{*}{$\begin{array}{c}\text { Age of } \\
\text { infant } \\
\text { months })\end{array}$} & \multirow{2}{*}{$\begin{array}{l}\text { Blood plate- } \\
\text { let count } \\
\text { (thousands) } \\
\text { per cmm. }\end{array}$} & \multicolumn{2}{|c|}{$\begin{array}{c}\text { A rakawa's } \\
\text { reaction }\end{array}$} & \multirow{2}{*}{$\begin{array}{l}\text { Increase } \\
\text { of blood } \\
\text { platelets }\end{array}$} \\
\hline & & & & & & Right & Left & \\
\hline 51 & U.I. & $\operatorname{Tan} \theta$ & 26 & 1.5 & 435 & $16(-$ & 15 & \\
\hline 52 & M.A. & Jan. & 27 & - $\quad 1.5$ & 430 & 15 & 16 & \\
\hline 53 & K.M. & Feb. & 35 & 15 & 430 & 14 & 16 ? & \\
\hline 54 & M.M. & Julyr & 25 & 4 & 430 & 16 & $16(-$ & . \\
\hline 55 & M.K. & April & 23 & 12 & 430 & 16 & 16 & $10^{\circ}$ \\
\hline 56 & M.S. & Jan. & 27 & 9 & 440 & 7 & 12 & 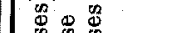 \\
\hline 57 & Y.I. & Dee. & 30 & 8 & 440 & 16 & 16 & 蛋㩊 = \\
\hline 58 & T.N. & Feb. & 26 & 10 & 440 & 16 & 16 & 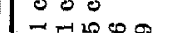 \\
\hline 59 & K.A. & June & 23 & 1.5 & 450 & 14 & 14 & $e^{-1} \mathrm{G}$ \\
\hline 60 & A.N. & Nov. & 27 & 2 & 450 & 8 & 16 & \\
\hline 61 & H.I. & July & 24 & 3 & 450 & 16 & 12( & 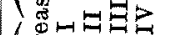 \\
\hline 62 & Y.Y. & Jan. & 24 & 1 & 450 & 12 ( & 16( & $\underbrace{0} \underbrace{-1 n-1-1}$ \\
\hline 63 & T.M. & 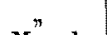 & 33 & 5 & 450 & 16( & 16( & a \\
\hline 64 & H.K. & March & 24 & 1 & 450 & 16( & 16 & $\rightleftarrows$ \\
\hline 65 & Y.S. & July & 25 & 11 & 460 . & 5 & & $8-$ \\
\hline 66 & J. T. & Sep. & 25 & 4 & 460 & 15 & & $\vec{d}$ \\
\hline 67 & C.E. & April & 32 & 8 & 470 & 9 & 9 & \\
\hline 68 & A.T. & $\operatorname{Jan}$. & 34 & 5 & 470 & 13( & 7 & \\
\hline 69 & M.E. & Feb. & 26 & 0.5 & 470 & 15( & 16 & 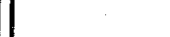 \\
\hline 70 & T.K. & June & 25 & 5 & 470 & 16 & 16 & \\
\hline 71 & Y.H. & Nov. & 24 & 9 & 470 & 16 & 16 & \\
\hline 72 & K.M. & Dec. & 32 & 4 & 470 & 16 & 16 & . \\
\hline 73 & I. K. & Jan. & 26 & 5 & 470 & 16 & 16 & \\
\hline 74 & F.S. & March & 25 & 1 & 480 & 11 & 2 & \\
\hline 75 & S. S. & Dec. & 35 & 15 & 480 & $3( \pm)$ & & \\
\hline 76 & S. A. & $\pi$ & 28 & 2 & 480 & $2(+)$ & 16 & \\
\hline 77 & $\mathbf{T}+\mathbf{F}^{2}$ & Oet. & 27 & 12 & 480 & 8 & & \\
\hline 78 & M.T. & Ang. & 86 & 9 & 480 & 11 & & \\
\hline 79 & X.C. & May & 25 & 5 & 480 & 16 & 150 & \\
\hline 80 & K.T. & Aug. & 27 & 2 & 480 & $16(-$ & 16 ( & \\
\hline 81 & M.K. & Nov. & 27 & 9 & 490 & $11( \pm)$ & & 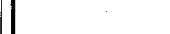 \\
\hline 82 & + M.I. & $\operatorname{Jan}$. & 23 & 9 & 490 . & 5 ( & & $\cdot$ \\
\hline 83 & M.M. & Dec. & 24 & 9 & 490 & 83 & 11( & \\
\hline 84 & T.T. & Jan. & 26 & 1 & 490 & 16 & 11( & . \\
\hline 85 & H.T. & April & 23 & 4 & 490 & 31 & 3 & \\
\hline 86 & T.A. & Nov: & 21 & 3 & 490 & 15 ( & 16( & \\
\hline 87 & M.A. & Jan. & 27 & 1.5 & 490 & 16( & 16 & \\
\hline 88 & $\mathbf{Y} . \mathbf{E}$ & $n$ & 25 & 3 & 500 & 16( & 14 & \\
\hline 89 & T.D. & Aug. & 25 & 3 & 500 & 7( & 14 & \\
\hline 90 & K.S. & March & 42 & 6 & 500 & 93 & $14( \pm$ & \\
\hline 91 & K.I. & Nov. & 29 & 3 & 500 & 16( & 16 & \\
\hline 92 & S. 0 . & Feb. & 30 & 2 & 500 & 16 & $16 ?$ & \\
\hline 93 & M.W. & June & 24 & 2 & 510 & $16(-\ldots)$ & 16 & \\
\hline 94 & T.S. & March & - 35 & 3 & 520 & $1( \pm)$ & $4^{\prime}$ & us \\
\hline 95 & M.I. & Jan. & 23 & 9 & 520 & $7 \pm)$ & & 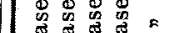 \\
\hline 96 & T.S. & Oet. & 27 & 10 & 530 & $4(+$ & $5(+4)$ & 马ु \\
\hline 97 & $\mathrm{~T} . \mathrm{F}$. & Sep. & 26 & 12 & 530 & $1(-$ & $4(-)$ & $\infty 0-\infty \infty$ \\
\hline 98 & S. H. & March & 26 & 2 & 540 & $9^{2}+3$ & $9( \pm)$ & $\approx \equiv$ \\
\hline 99 & S. F. & Jan. & 34 & 4 & 560 & $4(-)$ & $3( \pm)$ & \\
\hline 100 & H.S. & March & 28 & 2 & 570 & $12(-)$ & $11( \pm)$ & 怘司名 \\
\hline 101 & M.S. & Jan. & 28 & 9 & 570 & $16(-)$ & $14 \div$ & $0 \underbrace{\curvearrowleft ロ ロ \mapsto}$ \\
\hline 102 & K.o. & March & 24 & 1 & 590 & $16(-)$ & 16 & $.7 \underbrace{}_{0}$ \\
\hline 103 & H.O. & Dee. & 28 & 4 & 590 & $16(-)$ & $16(-)$ & 을 \\
\hline 104 & Y.N. & $\pi$ & 26 & $\mathbf{3}$ & 590 & $16(-)$ & $16 ?$ & 5 \\
\hline 105 & K.I. & Nov. & 24 & 3 & 600 & $16(-)$ & $16(-)$ & ). \\
\hline
\end{tabular}




\begin{tabular}{|c|c|c|c|c|c|c|c|c|}
\hline \multirow{2}{*}{$\begin{array}{l}\text { Case } \\
\text { No. }\end{array}$} & \multirow{2}{*}{ Name } & \multirow{2}{*}{$\begin{array}{l}\text { Month } \\
\text { of } \\
\text { experi- } \\
\text { ment }\end{array}$} & \multirow{2}{*}{$\begin{array}{l}\text { Age of } \\
\text { mother } \\
\text { (years) }\end{array}$} & \multirow{2}{*}{$\begin{array}{l}\text { Age of } \\
\text { infant } \\
\text { months }\end{array}$} & \multirow{2}{*}{$\begin{array}{l}\text { Blood plate- } \\
\text { let count } \\
\text { (thousands) } \\
\text { per cmm. }\end{array}$} & \multicolumn{2}{|c|}{$\begin{array}{c}\text { Arakawa's } \\
\text { reaction }\end{array}$} & \multirow{2}{*}{$\begin{array}{l}\text { Increase } \\
\text { of blood } \\
\text { platelets }\end{array}$} \\
\hline & & & & & & Right & Left & \\
\hline 106 & T.T. & Sep. & 28 & 2 & 620 & $15(-)$ & $15(-)$ & 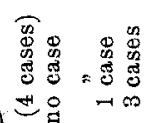 \\
\hline 107 & I. $\mathrm{H}$. & Jan. & 34 & 15 & 630 & $10 i-)$ & $7(+)$ & 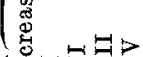 \\
\hline 108 & T.F. & Oct. & 27 & 12 & 640 & $14(-)$ & $13(-)$ & 5 \\
\hline 109 & T.A. & Jan. & 28 & 8 & 640 & $18(-)$ & $16(-)$ & 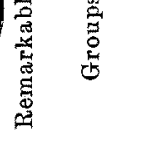 \\
\hline
\end{tabular}

TABLE 3.

Signs for showing different $A r a k$ a wa's reaction.

$$
1^{\prime} \quad 2^{\prime} \quad 3^{\prime} \quad 4^{\prime} \quad 5^{\prime}
$$

1. $1($ H) stands for +4 H H

2. $1(+t) \rightarrow$ H H H H H

3. $2(H) n$ H H $\#$ H

4. $3(H)$ \# $\#$ H H

5. $4(+4) \therefore \quad+4+4+H$

6. $5(H) \geqslant+H+H+H$

7. $1(+) \Rightarrow+H+H$ H

8. $2(+) \Rightarrow+H+H$ H

9. $3(t) \Rightarrow+t+H+H$

10. $4(+)$, + H H

11. $5(+) \quad+++$ H

12. $6(+),++\mathrm{H}+$ 州

13. $7(+),++H+H$

14. $8(+) \Rightarrow+++$ H

15. $9(+) \quad n+++H$

16. $10(+) \quad$ ++++ +

17. $11(+),++++$

18. 1(士) , $\pm+H$ H

19. $2( \pm) \quad$ $\pm+H+H$

20. $3( \pm) \quad \pm+H+H$

21. $4( \pm) \quad \Rightarrow+ \pm+4$

22. $5( \pm) \quad \Rightarrow \quad \pm++H+$

23. $6( \pm) \quad, \pm+++H$

24. $7( \pm) \quad$ $\quad \pm++++$ $\begin{array}{lllllll}1^{\prime} & 2^{\prime} & 3^{\prime} & 4^{\prime} & 5^{\prime}\end{array}$

25. $8( \pm)$ stands for $\pm \pm+H H$

26. $9( \pm), \quad \pm \pm+++$

27. $10( \pm) \quad$ $\pm \pm++H$

28. $11( \pm) \quad n \quad \pm \pm+++$

29. $12( \pm) \quad n \quad \pm \pm \pm++$

30, $13( \pm), \quad \pm \pm \pm++$

31. $14( \pm) \quad " \quad \pm \pm \pm \pm+$

32. $15( \pm), \quad \pm \pm \pm \pm \pm$

33. $1(-)$ - $\quad$ - $1+H$

34. $2(-) \quad n- \pm+$ H

35. $3(-) \quad n- \pm++H$

36. $4(-) \quad n- \pm++-1$

37. $5(-) \quad n- \pm \pm++$

38. $6(-) \quad n \quad \pm \pm++$

39. $7(-) \quad n- \pm \pm \pm+$

40. $8(-) \quad, \quad- \pm \pm \pm \pm$

41. $9(-) \quad \pi-- \pm+H$

42. $10(-) \quad$ - - \pm++

43. $11(-) \quad n,-\infty \pm+$

44. $12(-) \quad n \quad- \pm \pm \pm$

45. $13(-) \quad n \quad--- \pm+$

46. $14(-) \quad n \quad-- \pm \pm$

47. $15(-) \quad n--1$

Explanation to the Table:-

Take, for instance, the sign: $2(H)$. This stands for A rakawa's reaction with the course $(H) 1^{\prime}(H) 2^{\prime}(H) 3^{\prime}\left(H^{\prime}\right) 4^{\prime}(H) 5^{\prime}$. The sign does not express any prompt result of the reaction, so the prompt reaction of the sign: $2(H)$ may be $(-) 0^{\prime},( \pm) 0^{\prime}$, $(+) 0^{\prime}$ or even $(H) 0^{\prime}$, but this will not matter much, as the result of the reaction in one minute is the most important. 
TABLE 4.

Relation between A rakawa's reaction and blood platelet count of mothers taking vitamin $B$.

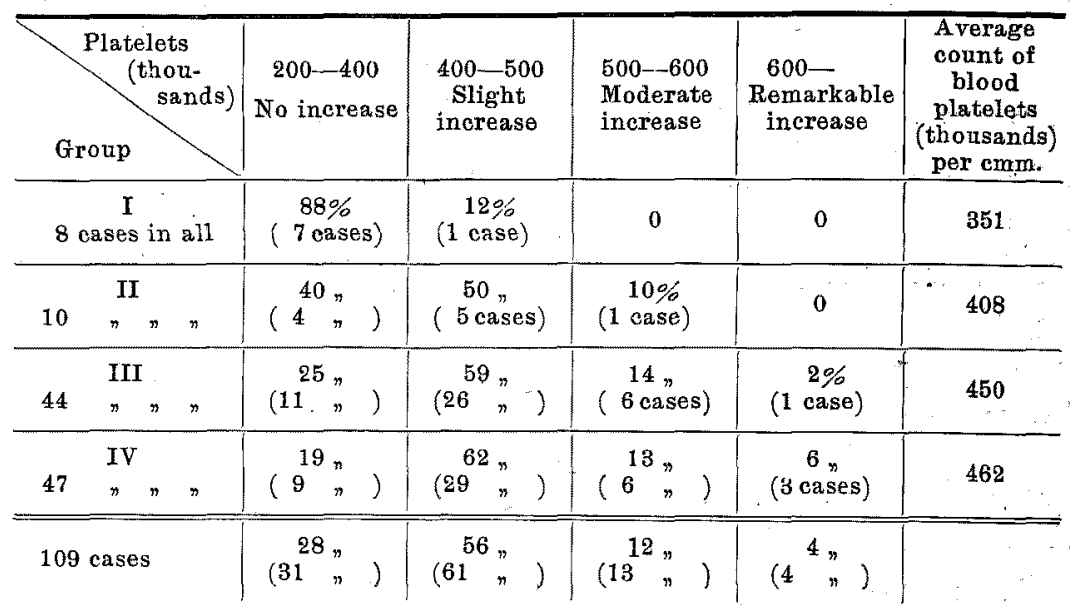

No case belonged to Group I or to Group II. 1 case belonged to group III and 3 cases to Group IV.

B) Classification according to Araka wa's reaction (Cf. Table 4).

1. Group I (Group of normal Arakawa's reaction): There were 8 cases out of 109 cases belonging to Group I. The platelet count showed no increase in 7 cases ( $88 \%$ ), and a slight increase in the remaining 1 case $(12 \%)$. There was no case of the count over 500 thousands. The platelet count of the cases belonging to Group I was 351 thousands on an average.

2. Group $\Pi$ (Group of better intermediate Arakawa's reaction): There were 10 cases out of $109^{\circ}$ cases belonging to Group II. The count showed no increase in 4 cases (40\%), a slight increase in 5 cases $(50 \%)$, and a moderate increase in the remaining 1 case $(10 \%)$. There was no case of the count over 600 thousands. The platelet count of the 10 cases was 408 thousands on an average.

3. Group ITI (Group of worse intermediate Arakawa's reaction): There were 44 cases out of 109 cases belonging to Group III. The count showed no increase in 11 cases (25\%), a slight increase in 26 cases (59\%), a moderate increase in 6 cases (14\%), and a remakable increase in 1 case ( $2 \%$ ). The average count was in these 44 cases 450 thousands. 
4. Group IV (Group of completely or almost completely negative A rakawa's reaction): There were 47 cases out of 109 cases belonging to Group IV. The count showed no increase in 9 cases (19\%), a slight increase in 29 cases (62\%), a moderate increase in 6 cases $(13 \%)$, and a remarkable increase in the remaining 3 cases $(6 \%)$. The count in these 47 cases was on an average 462 thousands.

\section{Comment.}

Those 109 lactating mothers in the present paper had been taking a vitamin B preparation on their doctor's advice, though they were "not suffering from beriberi".

From the reports published by $\mathrm{Id}{ }^{67} \mathrm{Kubo},{ }^{7)} \mathrm{Nakamura}{ }^{8)}$ and Iijima ${ }^{9)}$ it is ascertained that an increase of the blood platelet count is a symptom of beriberi or B-avitaminosis. From J. Kimura's ${ }^{2-4)}$ papers and from my own preceding paper ${ }^{13}$ we knew that there was an increase of the platelet count in the case of mothers with milk negative to Arakawa's reaction. That paper of mine showed further that there was a certain parallelism between the blood platelet count of lactating. mothers and the intensity of A rakawa's reaction,-a higher count and weaker reaction, or a lower count and stronger reaction. Now it will be interesting to know what is the relationship between Araka$\mathrm{w}$ a's reaction and the platelet count of those who had been taking a vitamin B preparation for a more or less long time.

As will be seen from Table 3 , the average count of blood platelets was 351 thousands in Group I of A raka wa's reaction, 408 thousands in Group II, 450 thousands in Group III, and 462 thousands in Group IV. Mothers showing an increase of the count were $12 \%$ in Group I, $60 \%$ in Group $I$, $75 \%$ in Group III, and $81 \%$ in Group IV.

Now, I will compare the present result with the result published in my preceding paper ${ }^{1)}$. The comparison is shown in Table 5 which is more simplified in Table 6 (Cf. Tables 5 and 6 ).

Let us look at Table 6 first. This will show that, if cases with both "no increase" and "slight increase" are combined together, then it is of the same influence upon blood platelet count whether mothers have been taking vitamin B or not (see later). But mothers with

6) Y. I d o, Fukuoka Ikwadaigaku Zassi. 1912, 6, 217.

7) T. K u bo, Tokyo Iji Sinsi, 1921, 1417.

8) M. Nakamura, Nippon Naikwagakkwai Zassi, 1925, 13, 317.

9) R. Iijim a, Rinsho Byorigaku Ketsuekigakkwai Zassi, 1935, 4, 531. 


\section{TABLE 5.}

Comparison of the platelet count between Arakawa-negative mothers taking no vitamin $B$ and ones taking the vitamin.

The percentage shows that of the cases.

\begin{tabular}{|c|c|c|c|c|c|c|c|c|}
\hline \multirow{2}{*}{$\begin{array}{l}\text { Degree of in- } \\
\text { crease of plate- } \\
\text { let count } \\
\text { Groups } \\
\text { of Ara- } \\
\text { kaw a's } \\
\text { reaction }\end{array}$} & \multicolumn{2}{|c|}{ No increase } & \multicolumn{2}{|c|}{ Slight increase } & \multicolumn{2}{|c|}{$\begin{array}{l}\text { Moderate } \\
\text { increase }\end{array}$} & \multicolumn{2}{|c|}{$\begin{array}{c}\text { Remarkable } \\
\text { increase }\end{array}$} \\
\hline & No $B_{k}$ & $\begin{array}{c}\text { Taking } \\
\mathbf{B}_{1}\end{array}$ & No $B_{1}$ & $\begin{array}{c}\text { Taking } \\
\mathbf{B}_{1}\end{array}$ & No $B_{1}$ & $\begin{array}{c}\text { Taking } \\
\mathrm{B}_{\mathrm{l}}\end{array}$ & No $B_{1}$ & $\underset{\mathrm{B}_{1}}{\text { Taking }}$ \\
\hline 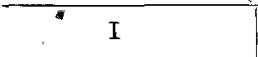 & $84 \%$ & $88 \%$ & $15 \%$ & $12 \%$ & $0 \%$ & $0 \%$ & $0 \%$ & $0 \%$ \\
\hline $\mathrm{II}^{*}$ & 43, & 40 & $28 \pi$ & 50 & $24 \%$ & $10 n$ & $5 n$ & 0 \\
\hline $\mathrm{III}$ & $25 \%$ & $25 n$ & 34 & 59 & $24 n$ & $14 n$ & $13_{n}$ & 2 \\
\hline$I V:$ : & 16 & 19 , & $36 n$ & 62 & $32 \%$ & $13 n$ & $16 n$ & 6 \\
\hline & $31 \%$ & 28 & $32 \%$ & 56 & $26 n$ & 12 & $11 \%$ & 4 \\
\hline
\end{tabular}

* This Group was shown as Groups II and III in my preceding paper.

$\begin{array}{lllllllll} & & & \end{array}$

TABLE 6.

Simplified comparison of the platelet count between Arakawanegative mothers with and without B-vitanin intake.

The percentage shows that of the cases.

\begin{tabular}{|c|c|c|c|c|c|}
\hline \multirow{2}{*}{$\begin{array}{l}\text { Groups } \\
\text { of Ara- } \\
\mathrm{kawa} \text { a's reaction }\end{array}$} & \multicolumn{2}{|c|}{$\begin{array}{l}\text { No increase and } \\
\text { slight increase }\end{array}$} & \multicolumn{2}{|c|}{$\begin{array}{c}\text { Moderate increase } \\
\text { and remarkable } \\
\text { increase }\end{array}$} & \multirow{2}{*}{$\begin{array}{l}\text { Average } \\
\text { count of } \\
\text { blood } \\
\text { platelets }\end{array}$} \\
\hline & $\begin{array}{l}\text { No } \\
\text { vitamin }\end{array}$ & Taken & $\begin{array}{c}\text { No } \\
\text { vitamin }\end{array}$ & Taken & \\
\hline I & $100 \%$ & $100 \%$ & $0 \%$ & $0 \%$ & 340 \\
\hline $\mathrm{II}^{*}$ & $71 \%$ & $90^{\prime \prime}$ & 29 n & $10_{n}$ & $\begin{array}{ll}431 & \\
& 408\end{array}$ \\
\hline III & $59 \%$ & $84 n$ & 37 & 16 , & $\begin{array}{ll}487 & \\
& 450\end{array}$ \\
\hline IV & 52, & $81 n$ & 37 & 19, & $504 \quad 462$ \\
\hline
\end{tabular}

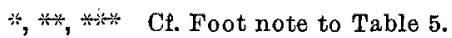

milk negative to Arakawa's reaction are, as Table 6 shows, of a smaller platelet count, if they have been taking the vitamin; or better, there is a smaller percentage of Arakawa-negative mothers taking the vitamin that are of a high platelet count than of those with no vitamin intake. 
This will also be seen from the average platelet count (Cf. Table 5), which is always smaller in the case of Arakawa-negative mothers (Groups II, III and IV) with vitamin intake than in the case of those with no vitamin intake $(408,450$ and 462 thousands as compared with 431, 487 and 504 thousands respectively).

In this regard, it is interesting to note that in Group I, the average platelet count is slightly larger in the case of mothers with the vitamin intake. The difference, however, is small, so that we may attribute the cause to a too small number of Arakawa-positive cases taking the vitamin ( 8 cases in all). But the following reason is very probably not to be excluded. The mothers treated of in the present paper began to take vitamin $\mathrm{B}$ on their doctor's advice, as stated above, so that these mothers, no Arakawa-positive, must have been Arakawanegative. Then it is not improbable that their platelet count must have been coming nearer and nearer the normal. At this time of writing, for instance, they may have shown a smaller count, At any rate, the average count is almost equal in Arakawa-positive mothers with or without vitamin intake.

If now we look at Table 5, then there is no remarkable difference between mothers with or without vitamin intake as to the distribution of cases according to the platelet count. The overwhelming majority of Group I belonged to "no increase". But as to the other Groups (II, III and IV), there is a much larger percentage of the cases with vitamin intake belonging to "slight increase" than those without it, while almost an equal percentage of both kinds of cases belonged to "no increase" (Cf. Table 5).

From the above, it will be clear that in Arakawa-negative mothers, blood platelet count is smaller in cases taking vitamin $B$, while in Arakawa-positive cases there is no remarkable difference whether they have been taking vitamin $\mathrm{B}$ or not, probably because the platelet count is or has come mostly within normal limits.

\section{Remarks.}

To prevent misunderstanding, I may briefly add that an intake of vitamin B may sometimes increase the blood platelet count. Suppose in a mother of whom we do not know anything of her A rakawa's reaction, we made a count and obtained the figures 250 thousands, and then 330 thousands,-say, after a week of vitamin B intake. It is of course not to be concluded that vitamin $B$ will increase the count 
generally, The mother may have been in a far advanced stage of avitaminosis $B$, and in such a case vitamin $B$ intake will increase the count and then only much later begin to decrease it, if the intake is continued longer.

\section{Summary.}

In the present paper I examined the blood platelet count of 109 apparently healthy lactating mothers with different A raka wa's reaction who had for a more or less long time been taking a vitamin B preparation.

The platelet count of 8 Arakawa-positive mothers (Group I) was within the normal limits in $88 \%$ of the cases, and only $12 \%$ showed a slight increase of the count. The count of Group II (the group having better intermediate A rakawa's reaction) showed a normal count in $40 \%$ of all 10 cases, a slight increase in $50 \%$, a moderate increase in $10 \%$. The count of Group III (the group of the worse intermediate A rakawa's reaction) showed a normal count in only $25 \%$ of all 44 cases, a slight increase in $59 \%$, a moderate increase in $14 \%$, and a. remarkable increase in 2\%. The count of Group IV (completely or almost completely negative A rakaw a's reaction) showed a normal count in only $19 \%$ of all 47 cases, a slight increase in $62 \%$, a moderate increase in $13 \%$, and a remarkable increase in $6 \%$. The average count was 351 thousands in Group I of A rak a wa's reaction, 408 thousands in Group $\Pi$, 450 thousands in Group $\Pi$ II, and 462 thousands in Group IV.

As I stated in my preceding paper, ${ }^{1)}$ there is a certain parallelisim between the blood platelet count of lactating mothers and the intensity of their Arakawa's reaction: Higher count and weaker reaction, or lower count and stronger reaction.

It is interesting to note the following about the average count in each group. In the groups (Groups II, III and IV) of cases with negative A rakawa's reaction, the average count is always smaller in the cases with vitamin $B$ intake than in those without it. But in the group of cases with positive Arakawa's reaction or in the group of cases with "good" or normal milk-the average count is about the same in the cases without vitamin $B$ intake as in the cases with it.

\section{Conclusions.}

In the present paper the relationship between the blood platelet. count and Arakawa-negative mothers taking a vitamin $B$ preparation. is shown. 
1. There is a certain parallelism between the blood platelet count of lactating mothers and the intensity of their Araka wa's reaction,the weaker the Arakawa's reaction, the higher the blood platelet count is.

2. In the groups of cases with negative Arakawa's reaction, the average count is always smaller in the cases with vitamin $B$ intake than in those without it. "But in the group of cases with positive Arakawa's reaction the average count is about the same in the cases without vitamin $B$ intake as in the cases with it. 